\title{
Integration of Renewable Energy Sources in Industrial Parks: Medicool European Demonstration Project
}

\author{
A. Molina-García ${ }^{1}$, T. García-Egea ${ }^{2}$ and M. Moreno ${ }^{3}$ \\ ${ }^{1}$ Department of Electrical Engineering - Campus Muralla del Mar \\ Universidad Politécnica de Cartagena - 30202 Cartagena (Spain) \\ Phone number: +34 986 325462, Fax number: +34 96832 5356, e-mail: angel.molina@upct.es \\ ${ }^{2}$ Polytechnic Science Department \\ UCAM Universidad Católica San Antonio de Murcia - 30107 Guadalupe-Murcia (Spain) \\ Phone number+34 96827000, email. tgarcia@ucam.edu \\ ${ }^{3}$ Medicool Engineering - Project Manager \\ HEFAME Group -Santomera-Abanilla 158, 30140 Santomera (Spain) \\ Phone number +3496827 7500, email. miguel.moreno@coiirm.es
}

\begin{abstract}
The present paper is focused on describing a demonstrative project, financially supported by the European Union, aiming of providing a real example of sustainable solution for space cooling in large storages and industrial applications. The proposed solution has been implemented in the pharmaceutical distribution sector, presenting strict and rigid European and national regulations regarding storage temperature conditions. The developed installation involves one of the largest systems ever built in Europe based on solar cooling/heating technologies. Collectors based on Ultra High Vacuum (UHV) technology have been selected, with a global collecting surface of $22,500 \mathrm{~m}^{2}$. These collectors also reduce significantly both convection and conduction heat losses. The proposed system involves two absorption chillers of 675 and $855 \mathrm{~kW}$ each one. An additional control system is included to fit the capacity of cold production according to the real necessities of the refrigerated system. This system is expected to provide an annual energy savings estimated in around 795 MWh only for the cooling system, accounting over $70 \%$ of the global energy currently needed for conditioning the global installations.
\end{abstract}

\section{Key words}

Solar cooling/heating system, solar energy

\section{Introduction}

During the last decades, energy use has become a crucial concern as a consequence of the rapid increase in energy demand. Moreover, environmental issues of conventional energy resources are continuously forcing us for alternative sources of energy, as a way of decreasing as climate changes as global warming. Moreover, conventional energy sources currently constitute around $80 \%$ of global energy consumption [1]. In this context, it is then desirable to promote renewable energy sources, such as solar, wind, biomass or hydropower, as promising $\mathrm{CO}_{2}$ free alternatives and real opportunities to decrease the conventional energy dependences. Indeed, different contributions have been focused on discussing the remarkable possibilities that solar energy is offering as a way of reducing significantly the power demand in the industrial sector [2][3]. In fact, and regarding the industrial sector, the necessities of cooling/heating power demand is increasing more and more in most developed countries. In moderate climates such as most EU member states, this power demand usually produces a dramatic increase of the electricity power demand during hot summer days, involving undesired increases of both fossil and nuclear energies and, furthermore, threaten the stability of electricity grids. These combined cooling/heating necessities are also becoming popular in residential and commercial sectors, for meeting desired thermal and electricity demands. From these requirements, solutions based on solar energy resources emerge as suitable alternative systems that integrate cooling/heating production and power generation capabilities. Additionally, these solar energy systems result in potentially lower capital and operating costs, facilitating maintenance and operation tasks [4]. Consequently, the application of renewable resources in general and solar solutions in particular for space heating/cooling purposes must be considered as a relevant topic of interest for the current industrial sector; mainly in countries such as Spain, where the climate offers suitable opportunities to integrate these solutions. Solar refrigeration is thus more and more recognized as a priority in developing countries, due to the needs for minimizing the energy expenditure and improvement the thermal requirements. 
In fact, the use of solar energy for absorption refrigeration research along this last decade has been one of the hot issues and scholars from all countries are studying deeply about it [5]. Actually, and according to recent contributions, refrigeration is particularly attractive as a solar energy application because of the near coincidence of peak cooling loads with the available solar power [6]. Under this scenario and considering the current cooling/heating necessities for the industrial sector as well as the incentives to promote the integration of renewable energy resources, the present paper is focused on describing a demonstrative project financially supported by the EU aiming of providing a real example of sustainable solution for cooling large storage where temperature and humidity requirements are very restrictive.

\section{The Medicool European Project}

\section{A. General Description}

The main objective of this innovative project is to evaluate a sustainable solution based on solar energy resources for cooling/heating large spaces with critical temperature and humidity requirements. This alternative solution aims to reduce significantly the power demand necessities, costs and greenhouse emissions. The Medicool project [7], financially supported by the European Union, is developed in a pharmaceutical installation located in Murcia, (South-East of Spain). This project has been focused on the pharmaceutical distribution sector, since it must fulfil rigid and critical European and national regulations in terms of storage conditions. Nowadays, and considering the current solutions based on conventional cooling/heating systems, these regulations are fulfilled in general at very high economic costs and with inefficient technical solutions, involving in some cases high environmental costs. The demonstration plant of solar cooling system, with a collecting surface of $22.500 \mathrm{~m}^{2}$, is one of the largest European systems, covering around $30,000 \mathrm{~m}^{2}$ of pharmaceutical installations. This centre provides services to hospitals and pharmacies in the Region of Murcia (Spain), supported by Hefame [8]. This is one of the three national leading companies in the sector of pharmaceutical distribution, including seven warehouses mainly in the Mediterranean regions of Spain. The installations must overcome significant drawbacks to maintain the indoor temperature within a narrow allowed interval around $25^{\circ} \mathrm{C}$. These limits are set by European and national regulations and codes for pharmaceutical products. In this specific case, the refrigeration problem is considered as a relevant issue by the regional public administration, since these pharmaceutical store installations are currently considered as external store centres by the public regional hospitals, which are not equipped with their own warehouse. Additionally, this remarkable interest in this project is also due to there are only a few projects devoted to assess and implement solar cooling/heating systems in real industrial parks and, most of them, propose smaller systems or prototypes not designed for the industry sector. Moreover, there are no examples of projects in Europe for thermal solar systems larger than $1 \mathrm{MW}$, being the most frequents systems below $100 \mathrm{~kW}$. Finally, it is necessary to point out that this solution is easily transferable to other economic sectors and industrial activities with similar characteristics and thermal requirements.

\section{B. Technical Innovations}

In reference to the innovate aspects of the proposed demonstrative project, new collectors with the latest technology have been selected, supposing one of the key elements of the system: the Ultra High Vacuum (UHV) collectors. These collectors reduce convection and conduction heat losses. Each collector is provided with a "Getter" pump that assures the vacuum. The collectors are composed by high absorbance $(>90 \%)$ and low emittance $(<7 \%)$ elements, which are exclusive for this kind of collectors and have not been provided by any other collectors. This system has been successfully tested at laboratory and pre-industrial level. These UHV collectors avoid evaporation problems of water by heating oil, reaching higher temperatures with lower pressure, which implies a simpler valve system. Evaporation is a major problem for this kind of installations, since the evaporation of water leads to the breakage of the collectors. In reference to the use of oil in the collectors instead of ordinary water presents several advantages based on the higher boiling point of oil: over $355^{\circ} \mathrm{C}$ at normal conditions. In the proposed prototype, UHV panels with a selective treatment of high absorbency and low emissivity collectors, provided with concentrators, have been selected. In these conditions, film temperature can reach higher values and, at these temperatures, exceptionally good thermal stability of the transmitting fluid is required. The proposed mineral oil has bulk temperatures up to $320^{\circ} \mathrm{C}$ and the rates of cracking and oxidation are very small, providing an efficient fluid heater with good pump circulation. Moreover, the use of oil decreases considerably the working pressure values, reducing then the exigencies and requirements of the piping, instrumentations and collectors. Due to the extremely high film temperatures in the collectors, the use of water would imply highpressure values with the aim of avoiding evaporation processes that may reduce the performance of the system.

The final implemented solution is a global system managing in real time, where the output temperature in each closed loop (126 components) is compared with the set-point value, modifying the input valve if it was necessary. Subsequently, the temperature is fitted according to the thermal requirements and the volume of oil changes with the available solar power. An additional regulation system is devoted to control the use of one or two absorption chillers. The system is thus in charge of deciding the suitable combination of machines and if they must be connected/disconnected according to the cooling/heating necessities and the availability of solar energy. Figure 1 and 2 show some details about the collecting surface as well as the installation process. 

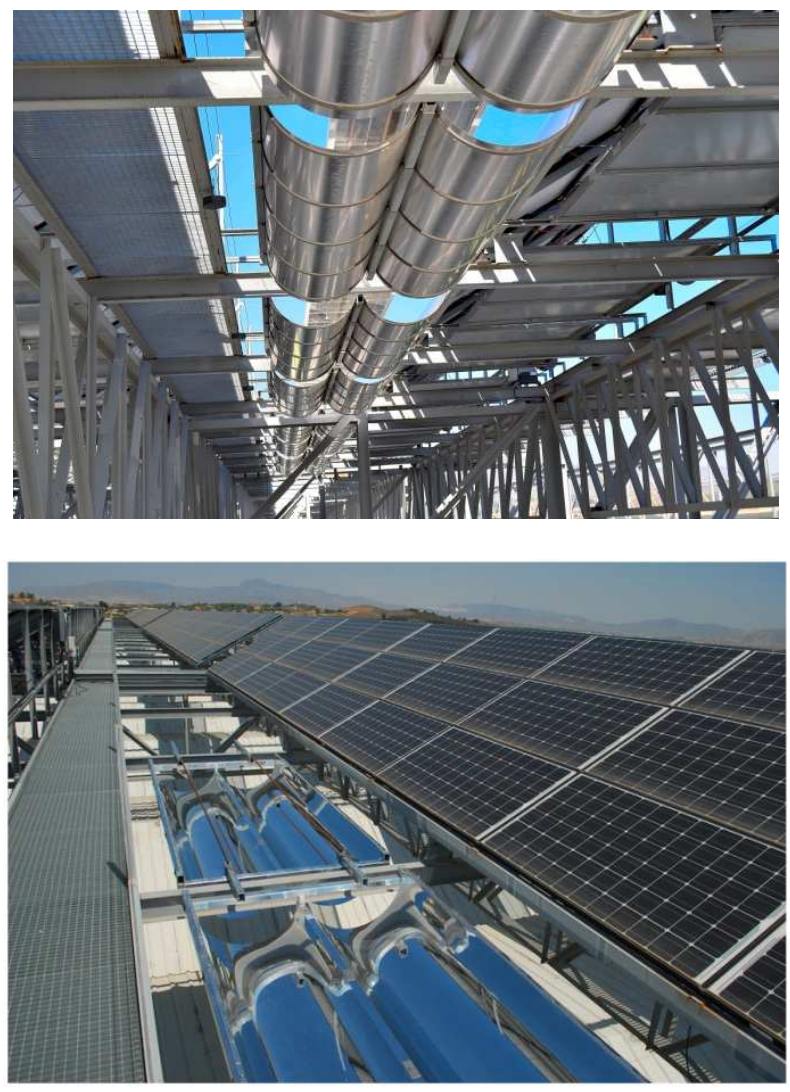

Fig. 1. Solar thermal cooling-heating system. General overview

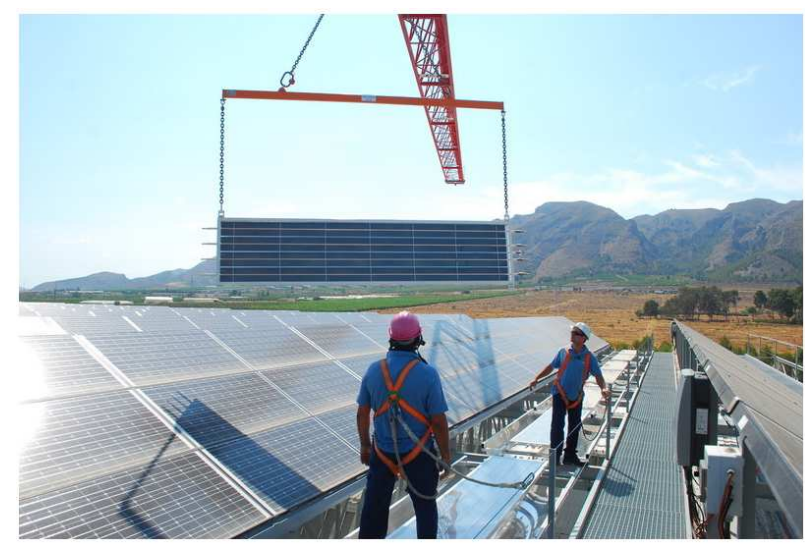

Fig. 2. Installation process and collecting surfaces

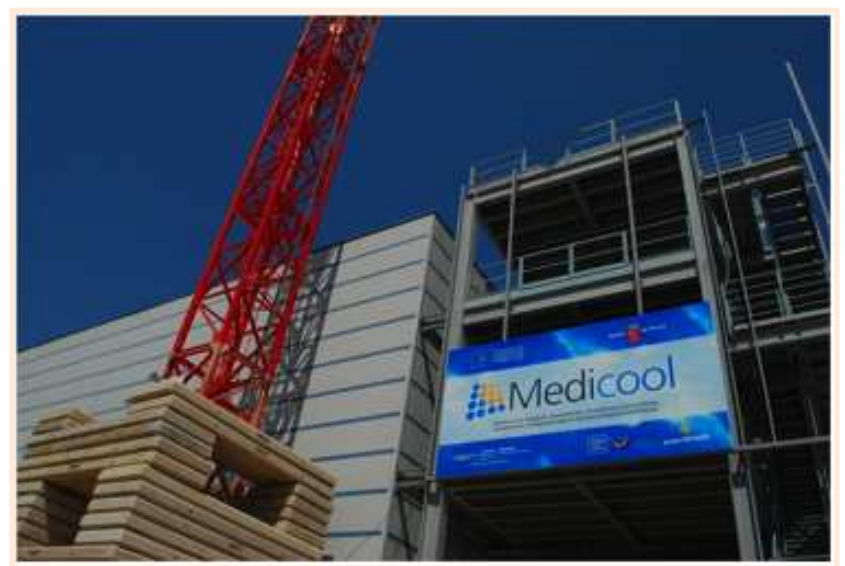

Fig. 3. Medicool demonstrative European Project

\section{Preliminary Results}

The proposed system is expected to provide an annual energy savings estimated in around $795 \mathrm{MWh}$ only for the cooling system, which represents more than $70 \%$ of the global energy currently needed for conditioning the warehouse. It means about $105 \mathrm{~m} €$, expected to increase according to the energy price modifications, and reaching up to $1.13 \mathrm{GWh}$ and $150 \mathrm{m€}$ if the heating energy savings in the offices were included. These energy reductions are even more relevant in daily and seasonally energy demand peaks, being the system more productive according to the energy demands are higher. In terms of $\mathrm{CO}_{2}$ savings, and considering a $\mathrm{CO}_{2}$ production of 0.233 $\mathrm{kg} / \mathrm{kWh}$ of $\mathrm{CO}_{2}$, the proposed thermal cooling/heating system is expected to save between 185 and 263 Tons of $\mathrm{CO}_{2}$ /year. Other types of emissions that can also be reduced are the following (per year): 766 Tons of $\mathrm{SO}_{2}$, 626 Tons of $\mathrm{NO}_{x}, 4140 \mathrm{~cm}^{3}$ of radioactive residues of low and medium activity, 508 gr. of high radioactive activity residues. At the same time, the implementation of this solar thermal technology in other warehouses with the same characteristics and the potential of transferability make these amounts to be much higher. The economic savings have to take into account indirect issues, as the decreases in electricity demand on peak power periods, especially in hot summer days. Figure 4 and 5 show respectively the flow heat exchanger values as a function of the estimated solar radiation data. As can be seen, significant power reduction can be achieved in comparison with conventional solutions as was previously discussed.

\section{Conclusion}

The European Project Medicool focused on providing and evaluating a real example of sustainable solution for cooling a pharmaceutical large industrial space is discussed and described in the present paper. The project includes one of the largest European systems based on solar cooling/heating technologies, with a collecting surface of 22,500 $\mathrm{m}^{2}$ with the main objective of reducing $795 \mathrm{MWh}$ due to cooling necessities, accounting over $70 \%$ of the global energy demand. It implies about 105 $m €$, expected to increase according to the energy price modifications, and reaching up to $1.13 \mathrm{GWh}$ and $150 \mathrm{m€}$ if the heating energy savings in the office buildings are included in a short-term.

The proposed system involves two absorption chillers of rate power of 675 and $855 \mathrm{~kW}$ respectively. For both absorption machines, an additional control system is included to fit the capacity of cold production according to the real cooling necessities of the pharmaceutical installations. A recent and innovate solution for the collector system is selected, supposing one of the key elements of the system: the Ultra High Vacuum (UHV) collectors. These collectors reduce significantly both convection and conduction heat losses. 


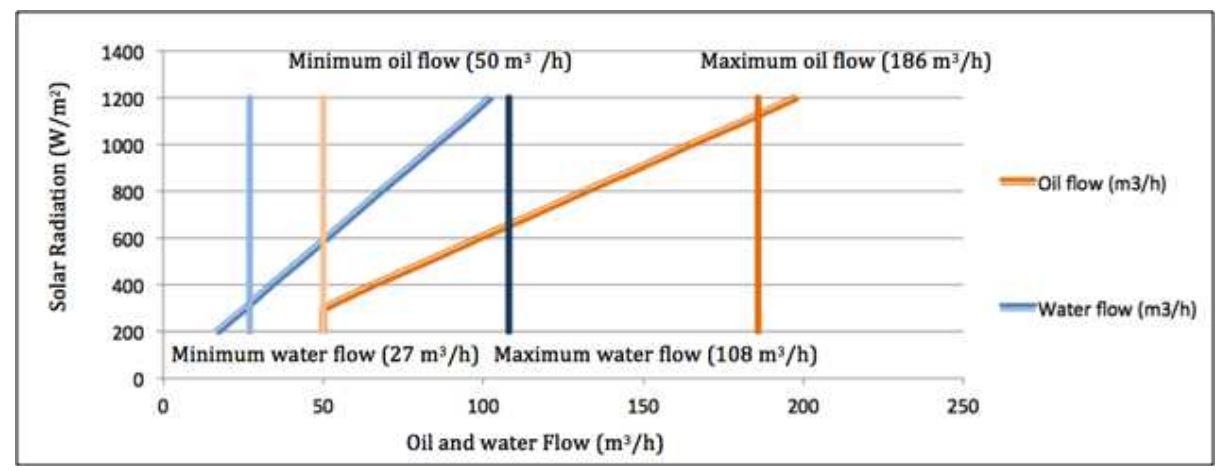

Fig. 4: Estimated oil and water flow absorption chiller. $675 \mathrm{~kW}$ nominal cooling capacity

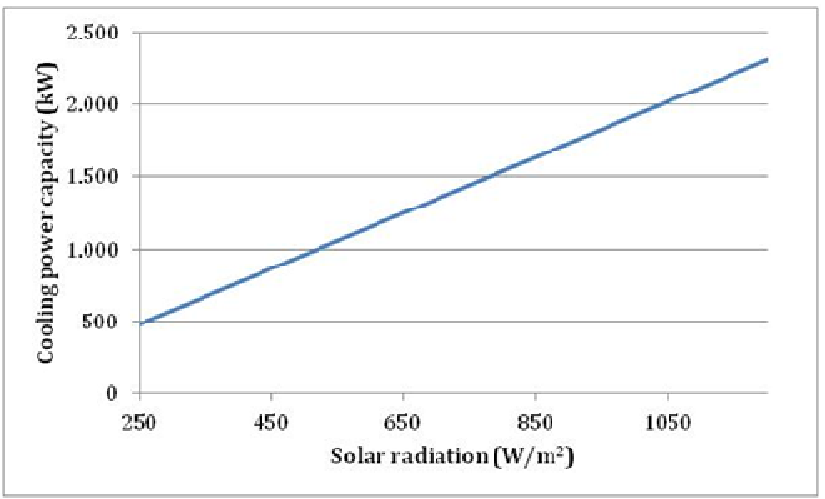

Fig. 5. Estimated cooling power capacity (kW)

The estimated energy reductions are even more relevant in daily and seasonally energy demand peaks, being the system more productive according to the energy demands are higher. In terms of $\mathrm{CO}_{2}$ savings, and considering a $\mathrm{CO}_{2}$ production of $0.233 \mathrm{Kg} / \mathrm{kWh}$ of $\mathrm{CO}_{2}$, the proposed thermal cooling/heating system is expected to save between 185 and 263 Tons of CO2/year. Other types of emissions that can also be reduced are the following (per year): 766 Tons of $\mathrm{SO}_{2}, 626$ Tons of $\mathrm{NO}_{\mathrm{x}}, 4140 \mathrm{~cm}_{3}$ of radioactive residues of low and medium activity, $508 \mathrm{~g}$ of high radioactive activity residues.

\section{Acknowledgement}

The LIFE+ European Program has financially supported this project. (Ref. LIFE10 ENV/ES/456).

\section{References}

[1] S. Mekhilef, R. Saidur, A. Safari, "A review on solar energy use in industries", Renewable and Sustainable Energy Reviews, vol. 15, 2011, pp. 1777-1790.

[2] H. Schnitzer, B. Christoph, G. Gwehenberger "Minimizing greenhouse gas emissions through the application of solar thermal energy in industrial processes. Approaching zero emissions", Journal of Cleaner Production, vol. 15, 2007, pp. $1271-1286$.

[3] E.F Bazen, M.A. Brown, "Feasibility of solar technology (photovoltaic) adoption: a case study on Tennessee's poultry industry", Renewable Energy, vol. 34, 2009, pp. 748-754.

[4] D.W. Wu, and R. Z. Wang, "Combined cooling, heating and power: a review" Progress in Energy and Combustion Science, vol. 32, 2006, pp. 459-495

[5] R.Z. Wang, M. Li, Y.X. Xu et al. "An energy efficient hybrid system of solar powered water heater and absorption ice maker", Solar Energy, vol. 68(2), 2000, pp. 189-195.

[6] K. Sumathy, K.H. Yeung, Li Yong "Technology development in the solar absorption refrigeration systems", Progress in Energy and Combustion Science, vol. 29, 2003, pp. 301-327

[7] Medicool. European project financed by the LIFE+ program (LIFE10ENV/ES/456), available on http://www.medicool.org

[8] HEFAME Group, available on http://www.hefame.es 